\title{
Una construcción histórico-epistemológica del modelo del octeto para el enlace químico
}

Resumen: En este artículo se presenta una reconstrucción histórico-epistemológica del modelo del octeto, para demostrar que el concepto de enlace químico se elaboró inicialmente como una representación icónica. El trabajo se realizó con profesores en formación avanzada, del programa de Maestría en Docencia de la Química, de la Universidad Pedagógica Nacional. Se pretendió avanzar en la problemática de la construcción de modelos científicos en el aula.

Palabras clave: Modelo científico, modelación, formación de profesores de ciencias.

\author{
Sulma Urbina Duarte* \\ Rómulo Gallego Badillo** \\ Royman Pérez Miranda** \\ Adriana Patricia Gallego Torres**** \\ Artículo recibido: 16-8-2006 y aprobado: 30-4-2008
}

A historical and epistemological construction of the octete model to the chemical bond

Abstract: In this paper the historical and epistemological construction of the octete model is presented in order to demonstrate that the concept of chemical bond was initially written as an iconical representation. This study was carried out with teachers that belong to the Magister program in Chemistry teaching at Pedagogica Nacional University. It was expected to advance in the scientific models construction for the classroom.

Key words: Scientific model, modeling, science teacher's education.

* Candidata a Magíster en Docencia de la Química. sulmau@starmedia.com.

** Profesores de la Universidad Pedagógica Nacional. Grupo de Investigación Representaciones y Conceptos Científicos, Grupo IREC.rgallego@uni.pedagogica.edu.co; royman@uni.pedagogica.edu.co.

*** Profesora de la Universidad Distrital Francisco José de Caldas. Adriana.P.Gallego@uv.es. 


\section{Introducción}

La construcción de modelos científicos en el aula, con estudiantes y con profesores de ciencias en formación, es uno de los nuevos campos de investigación en didáctica de las ciencias; campo este que se conoce como modelación o modelado. Inscritos en esta problemática, los directores del grupo IREC han adelantado un trabajo con profesores en formación avanzada y en formación inicial. El punto de partida fue la crítica que se hizo al empleo de la concepción de modelo de Giere (1990), en el sentido de que esta no puede ser aplicada a todas las ciencias de la naturaleza, en particular a la química, dada la diferencia en su construcción histórica con respecto a la de la física (Greca y Dos Santos, 2005).

Las revisiones histórico-epistemológicas permiten concluir que la química se constituyó como una de las ciencias de la naturaleza, siguiendo un lenguaje conceptual y metodológico distinto del seguido por los físicos. Este lenguaje, de carácter hipotético-deductivo, es el que ha adquirido pleno significado para la construcción y modificación paulatina de modelos icónicos o gráficos, mientras que en la física han predominado los modelos simbólicos. En consecuencia, para la elaboración de una didáctica de esta ciencia, es necesario aproximarse a la historia de esas construcciones y modificaciones, con miras a que profesores en formación avanzada y en formación inicial se introduzcan en una comprensión de cómo la comunidad de químicos le han dado a su actividad un estatuto científico particular.

\section{La categoría de modelo científico}

Muchos son los especialistas que se han ocupado de precisar esta categoría (Scheler, 1936; Hanson, 1977). Otra especialista que se ocupa de discernir sistemáticamente acerca de la categoría de modelo es la profesora Lombardi (1998), quien se adentra en la relación que existe entre los modelos de la física y los modelos matemáticos. Puntualiza que estos últimos se ocupan de los sistemas axiomáticos o conjunto de fórmulas, relacionadas deductivamente mediante una lógica subyacente y que el contenido semántico de un modelo matemático, se establece cuando se procede a darle una interpretación al sistema, asignándole a cada término una referencia que pertenece a un dominio previamente establecido. Aclara que no todo modelo científico tiene como referente el sistema real o natural del cual pretende dar cuenta. En el caso de la química, Tomasi (1999) clasifica los modelos en materiales y abstractos, y estos últimos, a su vez, en icónicos, analógicos y simbólicos. Subraya que los científicos establecen jerarquías de modelos o modelos de modelos, por exigencias de su actividad.

El modelo será icónico o gráfico, cuando el objeto de saber no es directamente accesible a una observación primera, no mediada instrumentalmente; una no mediación en virtud de que el estado de la investigación tecnológica no ha diseñado y construido los instrumentos requeridos. Afírmese que el modelo icónico que los químicos, a partir del modelo atómico de Dalton, 
fueron construyendo paulatinamente para representar la estructura molecular de los materiales, tuvo su contrastación positiva, primero con la síntesis de la urea y después con la de los azúcares existentes en la naturaleza, lo cual les indicó que era admisible. Luego, esa contrastación positiva se reforzó con la síntesis de la primera sustancia no existente en la naturaleza, la baquelita, a la que le siguió la de la sacarina.

El modelo será analógico cuando esas concepciones de partida tienen como fundamento un constructo técnico o tecnológico, mediante el cual se establece que las características e interacciones pueden ser representadas idealizando las propias de ese constructo. Podría señalarse al respecto la "mesa de billar" para el modelo cinético molecular de los gases y la máquina térmica ideal de Carnot para la termodinámica clásica. Este tipo de modelo fue introducido por los químicos, cuando acudieron a esta termodinámica para dar cuenta de la estabilidad de los materiales y la cinética y la dirección de los procesos químicos.

El modelo es simbólico cuando se reformula el analógico, en virtud de que la estructura conceptual y metodológica se transforma para ser un conjunto de expresiones matemáticas en términos de ecuaciones diferenciales lineales, en las que se recogen las leyes que gobiernan los fenómenos que existen, puesto que se han hecho objeto de estudio mediante un sistema y ese modelo. La introducción de los modelos simbólicos en la química se da a partir de los trabajos del grupo de Manchester, liderado por el profesor Tomson. Ocurrió con la explicación dada a los experimentos con los tubos de descarga, la reconsideración de las leyes electroquímicas de Faraday y el fenómeno de la radiactividad. La consecuencia fue el abandono definitivo del modelo icónico de Dalton. Los desarrollos posteriores que condujeron a la mecánica de matrices de Heisenberg, la cuantificación de la energía por Planck y la mecánica ondulatoria de Schrödinger, fueron la base para nuevas explicaciones del enlace químico: el enlace de valencia y el del orbital molecular.

No obstante la admisión de estos modelos simbólicos, los químicos, dentro de su objeto de conocimiento, cual es el de dar cuenta de la composición de la materialidad del mundo, de construir representaciones de esa composición, han puntualizado que ese objeto no es la atomicidad sino la molecularidad (Gallego Badillo, Pérez Miranda y Torres de Gallego, 1995; Hoffmann, 1997). Para la síntesis de nuevos materiales acuden a las representaciones icónicas, (Ihde, 1984), a partir de las cuales han demostrado que su ciencia, distinta de la física, es productiva y ha transformado el mundo de las relaciones entre los seres humanos. Hay, entonces, una justificación para una didáctica de la química fundamentada en la reconstrucción histórica de estos modelos icónicos o gráficos.

\section{Reconstrucción de modelos científicos y formación de profesores} La comunidad de especialistas en didáctica de las ciencias de la naturaleza ha venido ocupándose de la construcción en el aula de modelos científicos, tanto con profesores de ciencias en formación como con docentes universitarios de estos profesores (Galagovsky y AdúrizBravo, 2001; Justi, 2002; Oliva, Aragón, 
Bonat y Mateo, 2003; Islas y Pesa, 2003; 2004). Los resultados obtenidos hablan a favor de que las expectativas en estos procesos no sean enteramente satisfactorias. Una hipótesis trabajada en el Grupo IREC postula que esto podría deberse, entre otras causas, al hecho de que la educación habitual en ciencias ha estado restringida al dominio de una aproximación empiropositivista, en su reducción tecnicista.

Por un lado, esta aproximación epistemológica suele omitir la necesaria revisión histórica en el aula, y los procesos comunitarios que condujeron a la construcción de los modelos científicos que, por una razón u otra, son convertidos en contenidos curriculares. Por otro, tal reducción suele limitar esos contenidos a las definiciones de los conceptos científicos (Mosterín, 1978) y a la repetición de los algoritmos requeridos para la resolución de ejercicios de lápiz y papel y para la ejecución mecánica de prácticas de laboratorio. Estas definiciones y los algoritmos correspondientes son transmitidos verbalmente, a la espera de un aprendizaje memorístico.

$\mathrm{Al}$ aceptar estos resultados y las características del ejercicio habitual de los profesores de ciencias, en la Universidad Pedagógica Nacional, dentro del programa de Maestría en Docencia de la Química, los directores del Grupo IREC decidieron adentrarse en la didáctica de la modelación con un grupo de profesores de química en formación avanzada. Conscientes de que el objeto de saber y de investigación de los químicos ha sido delimitado acudiendo a la categoría de modelo icónico o gráfico, optaron por adelantar el presente trabajo de reconstrucción histórica en torno a la propues- ta del octeto para el enlace químico. Se tuvo como guía la recomendación de que acudir a la historia (Matthews, 1994) es una de las estrategias metodológicas que pueden rendir mejores frutos, cuando la intencionalidad es la de introducir a los profesores de química en formación avanzada en el problema didáctico de la reconstrucción de modelos científicos, en este caso el del octeto.

Para tal efecto, el proceso debía objetivarse mediante el sometimiento de lo elaborado a la comunidad de interesados en esta actividad. En este sentido y después de las respectivas consultas bibliográficas, el compromiso primero fue la redacción de un documento borrador, con miras a su revisión por parte de tres pares académicos. Luego de realizar las correcciones sugeridas por esos pares, la siguiente actividad fue la de presentar una sustentación pública, especialmente convocada para tal efecto; sustentación que fue igualmente evaluada después por los integrantes del Grupo IREC.

La orientación metodológica se centró en la identificación y caracterización de las concepciones elaboradas por estos profesores en formación avanzada en torno a modelo científico, la razón de sus clasificaciones, los fundamentos de la taxonomía de los mismos y en particular la de modelo químico o icónico; las concepciones históricas de las ciencias y la necesidad de estas para una didáctica de la modelación en química. Esas identificaciones y caracterizaciones se realizaron con base en las lecturas recomendadas, las cuales fueron objeto de análisis y discusión en el Grupo IREC.

De la misma manera se procedió con las lecturas de los originales disponibles sobre la génesis y desarrollo del 
modelo icónico del octeto. Las discusiones giraron en torno a, primero, las versiones lineales de esa reconstrucción y, segundo, el potencial didáctico de la misma en el trabajo con profesores en formación inicial y con estudiantes de educación media.

\section{La construcción histórica del modelo del octeto}

El primer modelo icónico para el átomo de los químicos lo propone Dalton, en su A new sistem of chemical phylosphy, publicada en 1807, específicamente en los capítulos "Acerca de la constitución de los cuerpos" y en "Acerca de la composición química”. Dalton representa los átomos con esferas y círculos, introduciendo puntos y rayas para diferenciar las distintas sustancias simples y compuestas identificadas en su tiempo. Desde su modelo atómico, Dalton promulga la Ley de las proporciones múltiples, al mismo tiempo que Gay-Lussac, la de los volúmenes de combinación y Proust, la de las proporciones definidas o constantes. Anótese que Dalton no aceptó la de los volúmenes de combinación. Estas leyes y el modelo icónico de Dalton fueron admitidos por Berzelius (Lockemann, 1960), el químico y profesor más influyente de la época.

Para explicar la extraña aritmética química de la ley de los volúmenes de combinación, Avogadro, en 1811, formula la hipótesis que lleva su nombre; hipótesis que tardó varios años en ser admitida por la comunidad de especialistas (Lockemann, 1960). Laurent, basándose en Avogadro, define los conceptos de peso molecular, molécula $y$ átomo. En este contexto de análisis y reconceptualizaciones, Wichelhaus pro- pone el concepto de valencia para denominar la atomicidad, en las discusiones sobre la estructura de los compuestos orgánicos. Frankland, en 1852, recoge el concepto de valencia y lo apuntala desde las leyes de las proporciones definidas y múltiples (Gallego Badillo, Pérez Miranda, Uribe Beltrán, Cuéllar Fernández y Amador Rodríguez, 2005).

Dentro de esta problemática conceptual y metodológica, A. Kekulé, en 1857, se decidió por la tetravalencia del carbono y defendió el modelo de las cadenas de átomos de carbono que había propuesto F. Rochleder, en 1852. A. Bluterov crea el concepto de estructura molecular (Lockemann, 1960). A. S. Couper fue el primero en representar las cuatro "afinidades del carbono" mediante una representación gráfica, en la que une cada carbono con una línea, dándole concreción a la representación estructural de las moléculas orgánicas (Schneer, 1975). Como consecuencia de estos desarrollos, los químicos reformularon paulatinamente el modelo icónico inicial de Dalton; reformulación que los conduciría a la síntesis de las moléculas existentes en la naturaleza y al diseño y fabricación en el laboratorio de nuevos materiales. La representación icónica modificada, como forma de comprensión e intervención en una materialidad no observable a simple vista, se estableció como un recurso conceptual y metodológico consensuado dentro de esta ciencia (Leidler, 1995).

Por su parte, los físicos entran a ocuparse del problema de la estructura última de la materialidad, específicamente de la continuidad o discontinuidad de la constitución de la misma. Teniendo como base la electrodinámica, los pro- 
yectos concentraron en los denominados rayos catódicos; los análisis de los datos obtenidos hablaron en favor de que estaban constituidos por unidades; unidades estas de carga negativa, que fueron deducidas del experimento de la gota de aceite de Millikan, en 1913. Es con estas conclusiones que se reconsideran los resultados electroquímicos de M. Faraday; por lo que el modelo atómico de Dalton comenzó a ser desplazado por las elaboraciones de los físicos (Uribe Beltrán y Cuéllar Fernández, 2003). Para Faraday, la idea de átomo era meramente metafísica (Schneer, 1975).

A esas unidades de masa y de carga eléctrica negativa, Stoney (1894) les dará el nombre de electrón. El electrón no se descubre como algo existente per se en la naturaleza; es una conclusión necesaria de unos resultados experimentales, leídos desde un modelo simbólico. Unas suposiciones análogas tendrán conclusiones semejantes con los denominados rayos canales, sobre los cuales se postulará la existencia de unidades eléctricas de carga positiva. Con base en los análisis e interpretaciones de los resultados de los tubos de descarga, Thomson (1904) propuso un modelo de átomo, el del pudín con pasas, que su equipo de trabajo buscó contrastar empíricamente con el experimento de la dispersión de partículas alfa, realizado por Geiger y Marsden (1909; 1913). Los resultados demostraron que este modelo era inconsistente. El análisis matemático que hizo Rutherford (1911) de esta dispersión le permitió proponer el modelo nuclear, el cual a su vez fue sustituido por el semicuántico de Bohr (1913).

Como consecuencia de los desarrollos anteriores, desde 1907 comienza a hacer carrera la idea de que la "compartición de electrones" por dos átomos es la que produce la unión entre ellos. Sir William Ramsay, quien identificó varios de los gases nobles, apoyó esta idea en 1908, y afirmó que los electrones sirven como "lazo de unión" entre átomo y átomo (Ihde, 1984). Siguiendo esta afirmación, por la misma época Johannes Stark, en su libro Die Elektrizität im chemischen Atom, consideró que los electrones de valencia atraían simultáneamente las partes positivas de dos átomos diferentes y elaboró una representación gráfica para ilustrar su punto de vista:

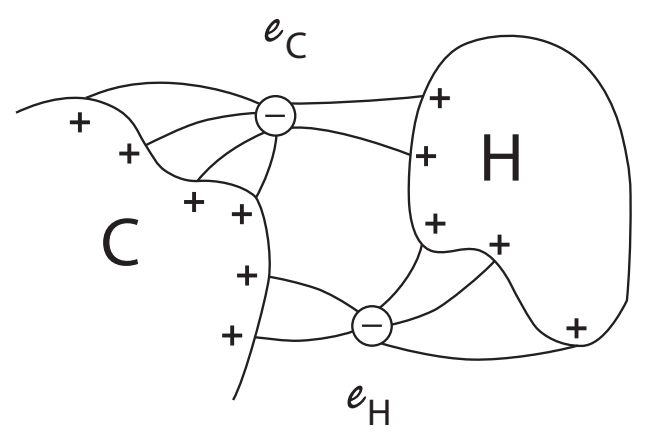

Figura 1. Modelo gráfico de Stark del enlace Carbono-Hidrógeno.

Para Stark, los electrones situados entre dos átomos constituyen el enlace químico. En consecuencia, propuso estructuras para varias moléculas. Así, en su representación del enlace $\mathrm{H}-\mathrm{C}$, tanto el electrón correspondiente al carbono como al del hidrógeno, enviaban líneas de fuerza a ambos núcleos, que servían de agentes enlazantes. En cuanto al amoníaco, supuso al nitrógeno con tres electrones de valencia y al hidrógeno con uno, como se muestra a continuación: 


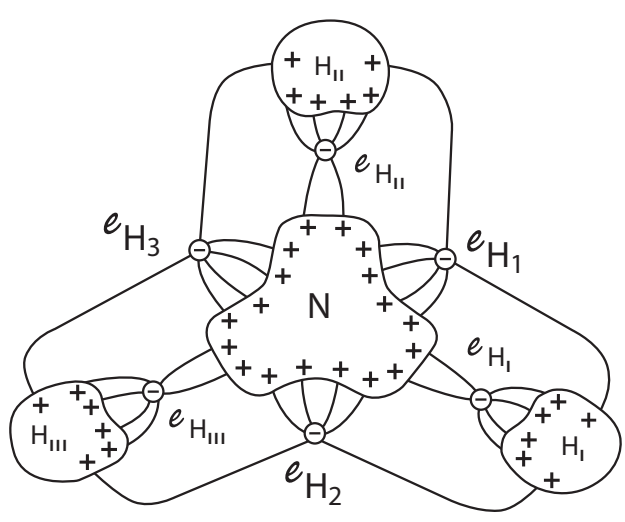

Figura 2. Modelo icónico de Stark para la molécula del amoníaco.

El modelo de Stark tuvo poca trascendencia. No así el de Bohr (1913). El científico danés propuso una representación icónica para la estructura electrónica de sistemas moleculares, con el fin de dar a conocer aquello que había concluido sobre los fenómenos electrónicos implicados cuando se formaba un enlace químico. Hizo dibujos tentativos de lo que pensaba sobre las estructuras del $\mathrm{H}_{2}, \mathrm{O}_{2}, \mathrm{H}_{2} \mathrm{O}, \mathrm{CH}_{4}$ y $\mathrm{C}_{2} \mathrm{H}_{2}$, aun cuando solo discutió en detalle la del hidrógeno, que se supuso constituida por dos núcleos positivos unidos por un anillo de dos electrones, así:
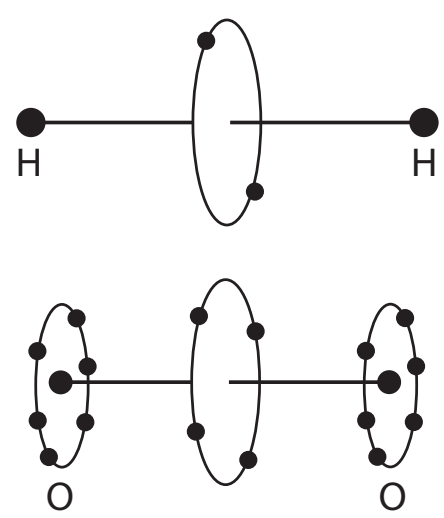

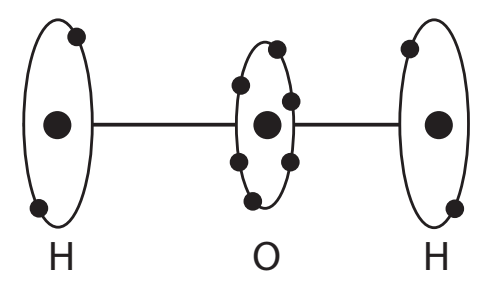

Figura 3. Modelos gráficos de Bohr (1913) para el hidrógeno, el oxígeno y el agua.

En su propuesta, supuso que el enlace químico era representable por un anillo de electrones que se movía en un plano perpendicular al eje internuclear. Para el hidrógeno, Bohr demostró su gran estabilidad y calculó su calor de formación en $264 \mathrm{KJ} / \mathrm{mol}$. Según este modelo (semicuántico), los electrones giran alrededor del núcleo, ubicándose en órbitas sucesivas que solo pueden tener un número limitado de electrones para formar enlaces; número dado por la expresión $2 \mathrm{n} 2$, siendo $n$ el número de la órbita. En el caso del litio con tres electrones, dos de ellos podrán ocupar la primera órbita $(n=1)$, pero el tercero deberá ubicarse en la segunda órbita de mayor energía. Entonces, este último electrón, al ser mucho menos atraído por el núcleo, puede ser removido para dar el ión $\mathrm{Li}^{+}$. Inversamente, un átomo de flúor, que tiene nueve electrones, podrá captar un décimo electrón a fin de completar su segunda órbita. Se tendrá entonces el ión F- En consecuencia, estos dos iones podrán formar un enlace electrovalente (o iónico) y producir el fluoruro de litio $\mathrm{Li}^{+} \mathrm{F}$.

Hay que precisar que el primer modelo electrónico de valencia fue propuesto por Abegg (1904), en el que sugirió que la estructura electrónica de los recientemente identificados gases nobles era la 
que les confería su estabilidad química y que para muchos de los otros elementos la suma de sus valencias positivas y negativas tenía que ser necesariamente ocho (Leidler, 1995). Destacó que la valencia positiva para cada elemento se correspondía con la posición de este en la tabla periódica.

En 1916 se publican dos importantes artículos sobre el modelo electrónico de valencia. El primero, de la autoría de Kossel (1916) y el segundo, de Lewis (Leidler, 1995). Kossel sugirió que cada elemento sucesivo contiene un electrón y una partícula positiva más que su predecesor. Concluyó que el cambio periódico en la valencia es una prueba de que al pasar de un elemento de menor peso atómico a otro de mayor peso atómico, la configuración electrónica cambia uniformemente. Por tanto, cada cierto recorrido por período se alcanzan configuraciones, para las cuales el número de electrones de valencia se repite; configuraciones que habría que asociar con los elementos químicamente inertes, corroborándose la ley de periodicidad de Mendeléiev (Bensaude-Vincent, 1991).

El concepto de valencia se constituyó como esencial para dar cuenta del comportamiento de los electrones externos de un átomo. Al igual que Bohr, Kossel concibe los electrones localizados en anillos concéntricos, a los electrones de un átomo rotando en órbitas alrededor del núcleo. A diferencia de Bohr, pensó que tanto el neón como el argón tenían ocho electrones en la órbita más externa, como lo representó en la siguiente figura, aplicable solo a los primeros 23 elementos.

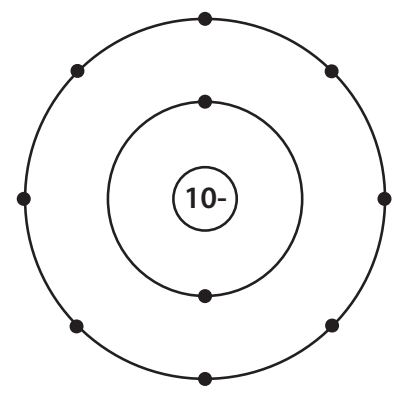

Figura 4. Modelo gráfico de Kossel (1916) para el neón.

Para Kossel, dada la relativa estabilidad química de los gases nobles, y que estos estaban precedidos en la tabla periódica por los halógenos (con un electrón menos) y seguidos por los metales alcalinos (con uno más), la transferencia de un electrón del metal alcalino al halógeno conducía a ambos a adquirir el mismo número de electrones que el gas noble correspondiente, lo cual era una razón para explicar la estabilidad de estos compuestos. Con ello, el halógeno adquiriría una carga negativa neta y el metal, una positiva. La atracción electrostática entre ambos iones sería responsable de la formación del enlace químico.

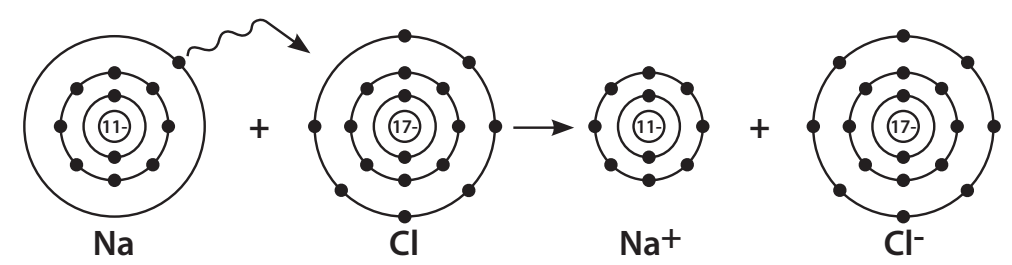

Figura 5. Modelo gráfico para la formación del cloruro de sodio, según Kossel (1916). 
Hay que destacar que este científico fue quien introdujo el concepto de transferencia de electrones de un átomo a otro, la cual forma iones con estructura de gas noble; idea que después Langmuir denominaría "electrovalencia" (Ihde, 1984). Para moléculas formadas por átomos iguales, Kossel (1916) no sugirió la transferencia de electrones, dado que para él ambos átomos presentaban la misma afinidad. Desde esta consideración acepta las ideas de Bohr, y postula también la presencia de anillos perpendiculares al eje del enlace. Para $\mathrm{H}_{2}, \mathrm{O}_{2}$ y $\mathrm{N}_{2}$ sugiere anillos con dos, cuatro y seis electrones, respectivamente.

Por su parte, Lewis (1916) propuso otro modelo, en el que los átomos adquirían, al combinarse, la estructura electrónica estable de los gases nobles. En lugar de la transferencia de electrones que había propuesto Kossel, Lewis afirmó que esa estabilidad se debía al compartimiento de un par de electrones; afirmación que constituyó uno de sus aportes al desarrollo de las ciencias. En su modelo, los electrones eran estáticos y arreglados en capas. La primera contenía dos electrones, mientras que las otras capas tenderían a contener ocho, a excepción de la última (capa de valencia), que contendría entre uno y ocho electrones (Bachelard, 1976); idea sobre los electrones estáticos que los físicos no admitieron (Leidler, 1995). Los primeros bosquejos acerca de su modelo fueron presentados por Lewis a sus estudiantes en 1902, cuando era instructor en la Universidad de Harvard (Leidler, 1995), por lo que puede deducirse que tuvo su origen en una intencionalidad didáctica.
El modelo electrónico de Lewis para la valencia, además de basarse en la poca reactividad de los gases nobles, tuvo como antecedente la Ley de Abegg, (1904) de valencia y contravalencia. En esa ley indicó que la diferencia entre el número de valencias positivas y negativas o "números polares" de un elemento era, frecuentemente, de ocho y nunca más de ocho. Así, si un átomo puede ceder $n$ electrones, entonces debe poder aceptar 8- $n$ electrones, lo que es consistente con el hecho de que en su capa de valencia pueda albergar hasta ocho electrones. Lewis (1916) representó, entonces, los electrones como ubicados en los vértices de un cubo, por lo que llamó a su idea: teoría cúbica del átomo; representación de la que los físicos, de nuevo, se burlaron, pero que para los químicos sigue siendo necesaria, dentro de sus propósitos de construcción de nuevos materiales (Leidler, 1995).

El problema siguiente, para Lewis, fue el de cómo representar con su modelo icónico los distintos elementos químicos y la variedad de compuestos. Lo resolvió mediante los siguientes postulados:

1. En cada átomo existe una parte esencial que permanece inalterada en todos los cambios químicos, llamada kernel (en alemán, almendra). En adelante se usará el término core en el mismo sentido). En esta porción del átomo se encuentran el núcleo y los electrones más internos (electrones de core), los cuales no alcanzan a neutralizar la carga del nucleo. Por ello, el core tiene una carga neta positiva, que corresponde al número de la familia a la cual pertenece el elemento en la tabla periódica. 
2. Adicionalmente al core, el átomo posee una capa externa que en el caso de un átomo neutro contiene la cantidad de electrones que determinan el comportamiento químico del átomo y se denominan electrones de valencia; electrones necesarios para neutralizar la carga positiva del core. Sin embargo, durante un cambio químico, los electrones, en esta capa, pueden variar entre cero y ocho.

3. En una combinación química, el átomo tiende a poseer un número par de electrones en la capa externa (de valencia) y específicamente ocho, los cuales se disponen simétricamente en los vértices de un cubo.
4. Las capas de valencia de diferentes átomos son mutuamente interpenetrables.

5. Los electrones pueden moverse con cierta facilidad de una posición a otra en la capa de valencia. No obstante, existen ciertas restricciones para este movimiento, que son determinadas por la naturaleza del átomo mismo, así como por la de aquellos otros combinados con este.

6. Las fuerzas eléctricas entre las partículas que están muy cerca (como en el átomo) no obedecen a la ley de Coulomb.
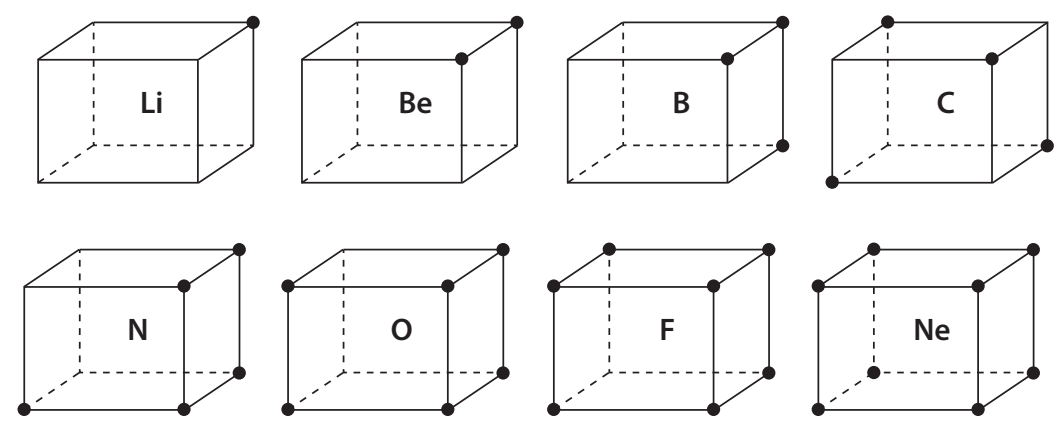

Figura 6. Modelo gráfico para los electrones de valencia, según G. N. Lewis (1923).

En su simbología, Lewis (1916) diferenciaba un elemento de su core positivo. Por ejemplo, Li representaba al core de un átomo de litio, o sea, el equivalente al ión $\mathrm{Li}^{+}$. Por ello, en la siguiente figura se representan los cores con negrillas. Como los átomos estaban constituidos por el core y los electrones de valencia (E), los átomos se representan de la siguiente manera:

$$
\begin{array}{lll}
\mathrm{Li} \Longrightarrow \mathrm{LiE} ; & \mathrm{Be} \Longrightarrow \mathrm{BeE} ; & \mathrm{B} \Longrightarrow \mathrm{BE}_{3} ; \\
\mathrm{C} \Longrightarrow \mathrm{CE}_{4} ; & \mathrm{N} \Longrightarrow \mathrm{NE}_{5} ; & \mathrm{O} \Longrightarrow \mathrm{OE}_{6} ; \\
\mathrm{F} \Longrightarrow \mathrm{FE}_{7} & &
\end{array}
$$

La representación $\mathrm{NE}_{8}$ correspondería al ión $\mathrm{N}^{-3}$. Lewis también empleó su simbología para representar compuestos. Colocó en sus fórmulas los cores de los átomos involucrados seguidos del número total de electrones de valencia. 
Por ejemplo:

$$
\begin{aligned}
& \mathrm{LiF} \Longrightarrow \mathrm{LiFE}_{8} ; \quad \mathrm{K}_{2} \mathrm{CO}_{3} \Longrightarrow \mathbf{K}_{\mathbf{2}} \mathrm{CO}_{3} \mathrm{E}_{24 i} ; \\
& \mathrm{C}_{2} \mathrm{H}_{2} \Longrightarrow \mathrm{C}_{2} \mathrm{H}_{2} \mathrm{E}_{10} ; \quad \mathrm{SO}_{4}^{-2} \Longrightarrow \mathbf{S O}_{4} \mathrm{E}_{3}
\end{aligned}
$$

Según el modelo icónico cúbico del átomo, algunas de las estructuras químicas que propuso fueron:

1. En la estructura del agua, únicamente el oxígeno ocuparía un cubo, ya que el hidrógeno puede tener, como máximo, dos electrones en su capa de valencia. Así:

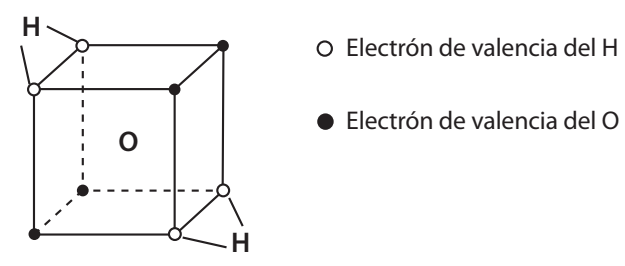

Figura 7. Modelo icónico de Lewis (1916), para la estructura del agua.

2. Para el $\mathrm{O}_{2}$ se formaría un doble enlace, compartido por cada átomo de oxígeno:

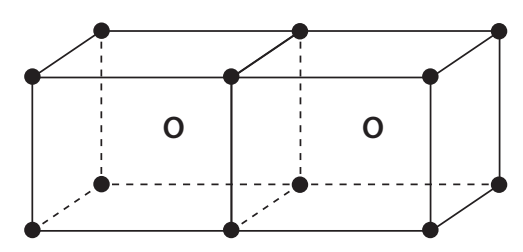

Figura 8. Modelo gráfico de (Lewis, 1916), para la molécula de oxígeno

3. En el tetracloruro de carbono, al compartirse un electrón de cada cloro con uno del carbono, ambos alcanzan una estructura de gas noble. Sin embargo, esta representación no satisface la naturaleza tetraédrica del carbono, ya postulado por A. Kekulé.

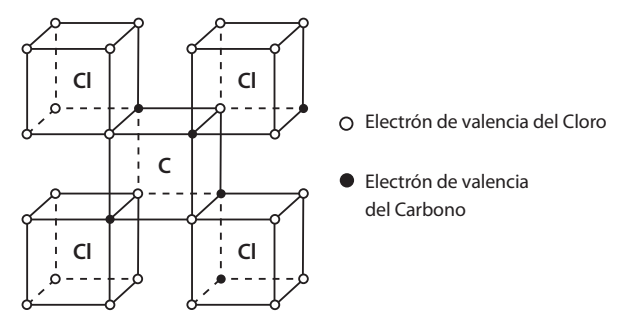

Figura 9. Modelo gráfico para el tetracloruro de carbono.

Por tal motivo, Lewis hace un arreglo diferente del grupo de los ocho electrones para el átomo de carbono, al cual se unen, quizás por fuerzas magnéticas, cuatro átomos o grupos de átomos.

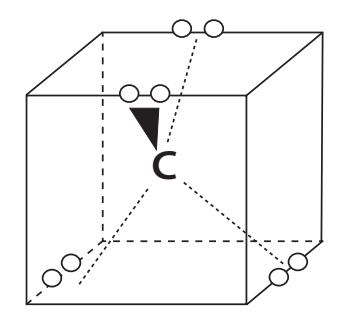

Figura 10. Modelo gráfico para el átomo de carbono (Lewis, 1916).

Finalmente, propone los conocidos diagramas o estructuras de puntos, en las que dos electrones ubicados entre dos átomos corresponden a un enlace sencillo. Asimismo, si el par electrónico está más cerca de un átomo que de otro, indica que la molécula es polar, en el que las líneas que se unen a los átomos están sobre ellos e implica la presencia de un par de electrones. 


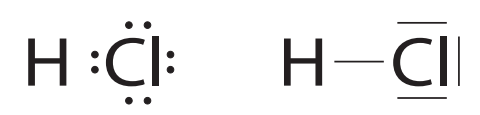

Figura 11. Modelo icónico para el ácido clorhídrico (Lewis, 1916).

Langmuir (1919) generalizó y extendió el trabajo de Kossel y de Lewis. A partir de los modelos de esto científicos, Langmuir formula cuatro postulados para predecir la reactividad química, la estructura y las propiedades de los compuestos. Compartir un par de electrones (o más) entre dos átomos, lo denominó enlace covalente. Su propio modelo predice la formación de otro tipo de unión, producto de la atracción electrostática entre las cargas, al que llamó enlace electrovalente. Para los electrones externos, o de valencia, de un átomo en un compuesto químico, sus cuatro postulados fueron:

1. Los arreglos estables y simétricos de electrones corresponden a los gases inertes y están caracterizados por fuertes campos de fuerza interna (atracción) y débiles campos de fuerza externa (repulsión). A menor número atómico, más débil es el campo externo.

2. El arreglo más estable de electrones es el par en el átomo de helio.

3. La siguiente configuración más estable de electrones es la del octeto, el cual corresponde a un grupo de ocho electrones, como el que se encuentra en la segunda capa del átomo de neón. Cualquier otro átomo con número atómico menor de 20 y que tenga más de tres electrones en la capa externa, tenderá a ganar el número adecuado de electrones suficiente para adquirir su octeto.
4. Dos octetos pueden tener uno, dos y, en ocasiones, tres pares de electrones en común. Un octeto puede compartir uno, dos, tres o cuatro pares de electrones con uno, dos, tres o cuatro octetos.

Desde estas consideraciones, Langmuir elabora su modelo del octeto de valencia. Los átomos en un compuesto tenderán a arreglarse de tal forma que todos ellos adquieran una configuración estable, la que para átomos con $\mathrm{Z}>5$ corresponde al octeto. Así, con el número total de electrones de valencia de tantos octetos como sean posibles, para un sistema con dos átomos (A y B), la configuración estable es factible de tres formas, según el cuarto postulado:

a) Es necesario que el número total de electrones de valencia sea 14 (dos electrones menos que los dos octetos aislados); b) 15 (cuatro menos que en dos octetos), y c) o 10. Si e es el número total de electrones de valencia, entonces el número de electrones compartidos entre los dos octetos será de 8(2)-e, y el numero, $p$, de enlaces covalentes entre A y B será de $p=1 / 2[8(2)-e]$. La ecuación anterior puede generalizarse para un número " $\mathrm{n}$ " arbitrario de octetos, en términos de $p=1 / 2[8 n-e]$.

Según Langmuir, esta última ecuación suministra información sobre cómo pueden arreglarse los octetos en una molécula. Por ejemplo:

1. Para el $\mathrm{Cl}_{2} \mathrm{O}: n=3$ (números de octetos) y $p=20$ (siete electrones de valencia de cada cloro y seis para el oxígeno); aplicando la fórmula anterior, $p=$ $1 / 2[8(3)-20]=2$; es decir, se formarían 
dos enlaces sencillos y una estructura acorde con ello sería:

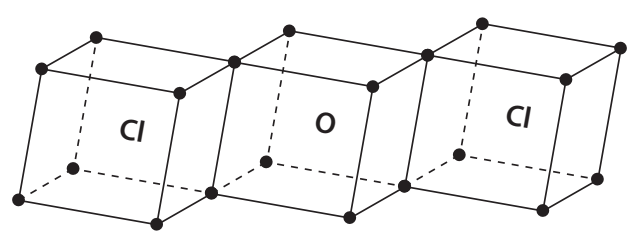

Figura 12

2. Para el $\mathrm{CO}_{2}, n=3, e=16$ y $p=1 / 2[8(3)$ $16]=4$; por tanto, puede proponerse entonces la existencia de dos dobles enlaces $\mathrm{C}=\mathrm{O}$. Empleando estructuras cúbicas, se obtiene:

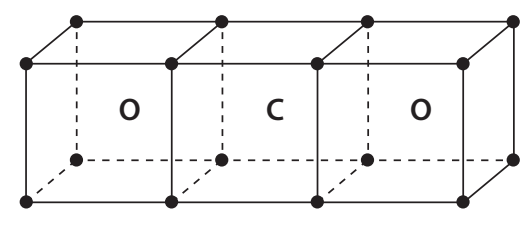

Figura 13.

Langmuir propone, también, una representación gráfica en la que los pares compartidos se encuentran más cerca entre sí:

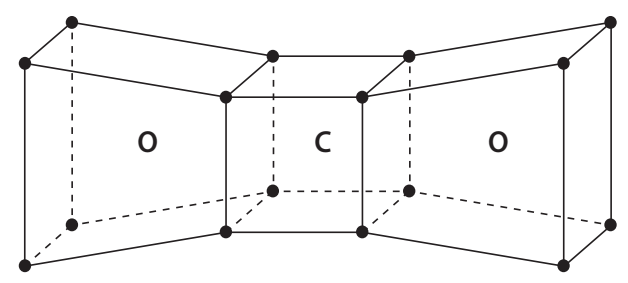

Figura 14.

El modelo de representación del enlace químico, acudiendo a la figura geométrica de cubos, por parte de Lewis (Ihde, 1984), con la concreción en el octeto de Langmuir, han sido y siguen siendo ampliamente utilizados para proponer las descripciones electrónicas y las estructuras de las moléculas. Todo porque el éxito de las mismas ha estribado en que, con ellas, los químicos han tenido la posibilidad de unir en un modelo icónico, mediante lazos inferenciales (Hanson, 1971), las evidencias experimentales de tipo químico, que de otra manera hubieran permanecido como hechos aislados.

Es preciso agregar que Bury, en 1921, propuso un esquema en el que, según las evidencias químicas, los electrones se distribuyen en capas sucesivas de 2 , 8,18 y 32 electrones. Este esquema fue reproducido por Sidwick, en 1927, en su libro The electronic theory of valency. Para este año y desde la mecánica ondulatoria, Heitler y London elaboraron el modelo del enlace de valencia, que fue desarrollada para moléculas más complejas que la del hidrogeno, por Pauling (1939). En 1929, Lennard-Jones, formuló el modelo del orbital molecular como una combinación lineal de los orbitales atómicos, conocido por sus siglas en inglés, como LCAO.

\section{Resultados}

De conformidad con las intencionalidades propuestas y en el campo restringido de la reconstrucción histórica del modelo icónico del octeto para el enlace químico, es posible afirmar que:

- La reconstrucción histórica adelantada, con base en el análisis crítico de la bibliografía de los originales disponibles, constituye un aceptable recurso didáctico para que los profe- 
sores en formación se aproximen al problema didáctico de la modelación o modelado.

- Esa aproximación no es del todo satisfactoria, en razón de que para los profesores en formación avanzada con los que se adelanto el proceso, la historia de las ciencias sigue siendo una ocupación paralela a la de trabajar en el aula cada modelo científico.

\section{Referencias}

Abegg, R. (1904). Die Valenz und das periodische Systeme. Zeitschrift. für anorganische Chemie, 39, 330-380.

Bachelard, G. (1976). El materialismo racional. Buenos Aires: Paidós.

Bensaude-Vincent, B. (1991b). Mendeléiev: historia de un descubrimiento. En: Historia de las ciencias, M. Serres (ed.), pp. 503-5025. Madrid: Cátedra.

Bohr, N. (1913). On the constitution of atoms and molecules. Philosophical Magazine, Series 6, vol. 26, 1-25.

Bury, C. R. (1921). Langmuir's theory of the arrangement of electrons in atoms and molecule. Journal American Chemical Society, 43, 1602-1609.

Galagovsky, y Adúriz-Bravo, A. (2001). Modelos y analogías en la enseñanza de las ciencias naturales. El concepto de modelo didáctico analógico. Enseñanza de las Ciencias, 19(2), 231-242.

Gallego Badillo, R., Pérez Miranda, R., Uribe Beltrán, M. V., Cuéllar Fernández, L. y Amador Rodríguez, Y. (2004). El concepto de valencia: su construcción histórica y epistemológica y la
- Predomina una versión lineal de la historia, de carácter empiropositivista, que se resiste a cambiar por una de carácter complejo.

- Es necesario que los investigadores en didáctica de las ciencias continúen allegando resultados confiables, para fundamentar conceptual y metodológicamente la didáctica de la modelación. $\boldsymbol{\Delta}$

importancia de su inclusión en la enseñanza. Ciência \& Educação, 10(3), 571-584.

Gallego Badillo, R., Pérez Miranda, R. y Torres de Gallego, L. N. (1995). La química como ciencia: una perspectiva constructivista. Química. Actualidad y Futuro, 5(1), 55-63.

Gieré, R. N. (1990). Explaining Science .Chicago: University of Chicago Press.

Greca, I. M. y Dos Santos, F. M. T. (2005). Dificuldades da generalização das estratégias de modelação em ciências: O caso da física e da química. Investigações em Ensino de Ciências. En línea:

http://www.if.ufrgs.br/public/ensino/vol10/n1/v10_n1_a2.htm

Hanson, N. R. (1971). Patrones de descubrimiento, observación y explicación. Madrid: Alianza.

Heitler, W. and London F. (1927). Wechselwirkung neutrale Atome und homopolare Bindung nach der QuantenmechanicK. Zeitschrift für Physik, 44, 455-472. 
Hoffmann, R. (1997). Lo mismo y no lo mismo. México: Fondo de Cultura Económica.

Ihde, A. J. (1984). The development of modern chemistry. New York: Dover.

Islas, S. M. y Pesa, M. A. (2003). ¿Qué rol asignan los profesores de física de nivel medio a los modelos científicos y a las actividades de modelado? Enseñanza de las Ciencias, número extra, 57-66.

Islas, S. M. y Pesa, M. A. (2004). Concepciones de los profesores sobre el rol de los modelos científicos en clases de física. Revista de Enseñanza de la Física, 17(1), 43-50.

Justi, R. S. (2002). Modelling Teachers' views on the Nature of modeling and implications for the education of modelers. International Journal of Science Education, 24(4), 369-387.

Kossel, W. (1916). Molecule formation as a question of atomic structure. Annalen der Physik, 49, 229-362.

Lennard-Jones, J. E. (1929). The electronic structures of some diatomic molecules. Transaction Faraday Society, 25, 668-686.

Leidler, K. J. (1995). The world of physical chemistry. Oxford: Oxford University Press.

Langmuir, I. (1919). The structure of atoms and the octet theory of valence. Proceedings of the National Academy of Science, vol. V, 252.

Lewis, G, N. (1916). The atom and the molecule. Journal of the American Chemical Society, vol. 38, 762 - 786.

Lewis, G, N. (1923). Valence and the structure of atoms and molecules. New York: Chemical Catalog Co.

Lockemann, G. (1960). Historia de la química. México: UTEHA.
Lombardi, O. (1998). La noción de modelo en ciencias. Educación en ciencias, II(4), 5-13.

Matthews, M. (1994). Science teaching: The role of history and philosophy of science. New York: Routledge.

Mosterin, J. 1978. La estructura de los conceptos científicos. Investigación y Ciencia, 16, 82-93

Oliva, J. M., Aragón, M. M., Bonat, M, y Mateo, J. (2003). Un estudio sobre el papel de las analogías en la construcción del modelo cinético-molecular de la materia. Enseñanza de las Ciencias, 21(3), 429-444.

Rutherford, E. (1911). The scattering of $\alpha$ and $\beta$ particles bay matter and the structure of the atom. Philosophical Magazine, Series 6, vol. 21, 669-688.

Scheler, M. (1936). Erkenntnis und Arbeit. Leipzig: Der neue Geist.

Schneer, C. J. (1975). Mente y materia. Barcelona: Bruguera.

Stoney, G. J. (894). Of the "Electron" or atom of etectricity. Philosophical Magazine, Series 5, vol. 38, 418-420.

Thomson, J. J. (1904). On the structure of the atom. Philosophical Magazine, Series 6, vol. 11, 769-781.

Tomasi, J. (1999). Towards chemical congruence of de model in theoretical chemistry. International Journal for Philosophy of Chemistry, 5(2), 79-115. En línea http://.hyle.org/journal/ issues/5-2/tomasi.html

Uribe Beltrán, M. V. y Cuéllar Fernández, L. (2003). Estudio histórico-epistemológico del modelo atómico de Rutherford. Tecné, Episteme y Didadxis, 14, 88-98. 